\title{
Best performing SiGe/Si core-shell nanoparticles synthesized in one step for high capacity anodes
}

Dr Antoine Desrues ${ }^{1}$, Dr John P. Alper ${ }^{1,2}$, Florent Boismain ${ }^{1}$, Diana Zapata Dominguez ${ }^{3}$, Dr Christopher Berhaut $^{4}$, Dr Pierre-Eugène Coulon ${ }^{5}$, Adrien Soloy ${ }^{1}$, Dr Frédéric Grisch ${ }^{6}$, Dr Samuel Tardif ${ }^{4}$, Dr Stéphanie Pouget ${ }^{3}$, Dr Sandrine Lyonnard ${ }^{4}$, Dr Cédric Haon ${ }^{2}$, Dr Nathalie Herlin-Boime*1

1 NIMBE, UMR 3685 CEA, CNRS, Université Paris-Saclay, CEA-Saclay, F-91191 Gif-sur-Yvette Cedex

2 CEA/LITEN/DEHT/STB/LM, F-38054 Grenoble Cedex 9, France

3 CEA/DRF/IRIG/DEPHY/MEM, F-38054 Grenoble Cedex 9, France

4 CEA/DRF/IRIG/DIESE/SyMMES, F-38054 Grenoble Cedex 9, France

5 LSI, CEA/DRF/IRAMIS, École polytechnique, CNRS, Institut Polytechnique de Paris, F-91128

Palaiseau

6 Normandie Univ., INSA Rouen, UNIROUEN, CNRS, CORIA, Rouen 76000, France
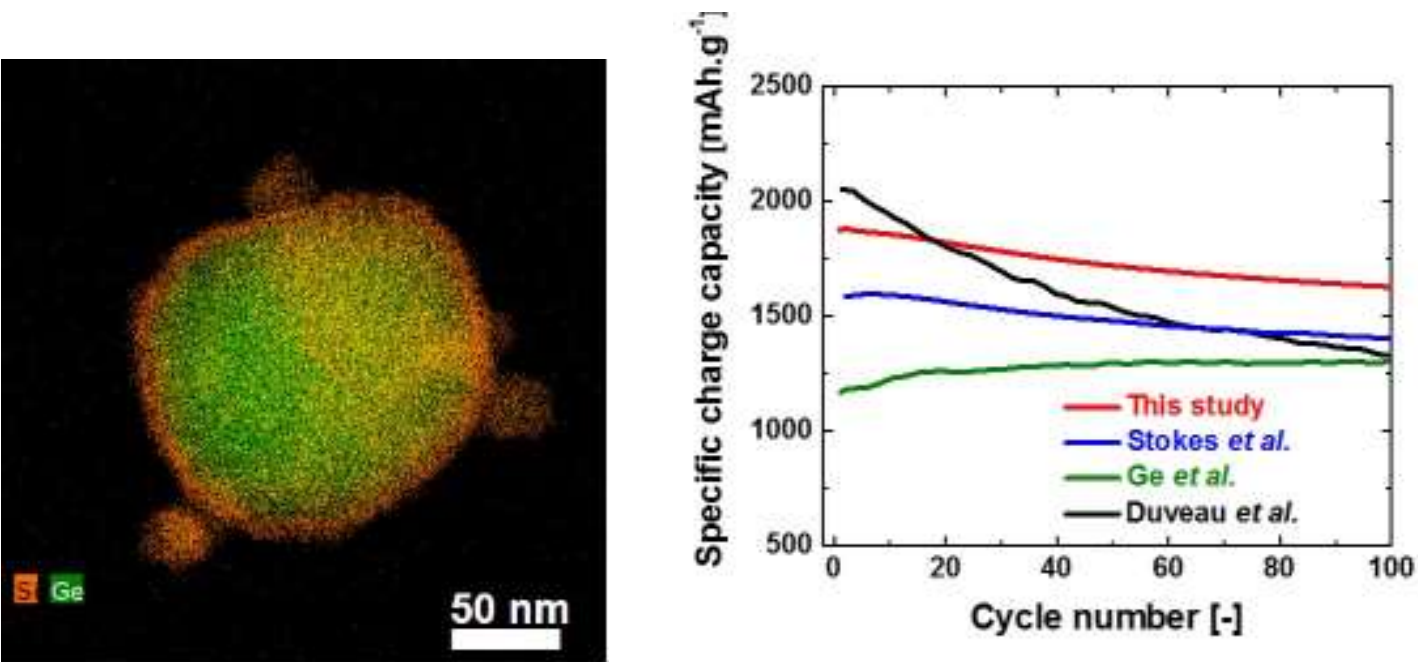

Graphical abstract

\section{Abstract}

Silicon-germanium nanostructures are promising anode materials for high stability, high capacity and fast cycling Li-ion batteries. In this work, we report on the outstanding performance of original $\mathrm{SiGe} / \mathrm{Si}$ core@shell nanoparticle heterostructures synthetized in one step by laser pyrolysis of silane and germane. By tuning the silane to germane ratio, the composition of $\mathrm{Si}_{100-x} \mathrm{Ge}_{x}$ alloy was readily adjusted. Nanoparticles with $x=0,20,47,77$, and 100 were investigated and the composition of each alloy (including internal mixed phases) was confirmed by $\mathrm{X}$-ray diffraction and energy-dispersive $\mathrm{X}$-ray spectroscopy. The electrochemical performances of the $\mathrm{Si}_{100-x} \mathrm{Ge}_{\mathrm{x}}$ alloys were evaluated by cycling half cell batteries from $\mathrm{C} / 5$ to $5 \mathrm{C}$. The optimal trade-off between stability and capacity was obtained in $\mathrm{Si}_{53} \mathrm{Ge}_{47}$ core shell nanoparticles alloy. This material exhibits the best performance reported so far for SiGe compounds, with a reversible specific capacity of 1695 mAh. $\mathrm{g}^{-1}$ after 60 cycles (90\% of its initial 
value). The (de)alloying properties of this optimal $\mathrm{Si}_{53} \mathrm{Ge}_{47}$ heterostructure were followed by Operando synchrotron WAXS measurements, suggesting sequential lithiation of the various phases present in the material. The alloying process, combined with the realization of peculiar nanostructures composed of a Ge-rich core and a Si-rich shell, therefore allow to reach electrochemical properties suited for a practical application in energy storage device.

\section{Introduction}

The development of electrochemical energy storage devices is considered key for achieving the energy transition and face the expected global increase in energy demand. A great challenge in this context is to improve the energy and power density, as well as safety and recyclability, of Li-ion batteries [1]. In the search for high capacity anode materials, silicon has attracted considerable attention due to its specific storage capacity, which is ten times higher than commercially employed graphite. However, the alloying process between lithium and silicon yields important volume changes on cycling (typically $280 \%$ for bulk silicon). The repeated sequences of expansion and contraction of the active silicon phase during successive lithiation and delithiation cause mechanical strains in the structure, fractures in the material, delamination of the electrode, permanent exposure of silicon to electrolyte and subsequent growth of an unstable Solid Electrolyte Interphase (SEI), leading to a rapid loss of performance [2]. Although strategies were developed to protect the silicon surface by carbon and, therefore, improve the stability [3-6], attempts to mitigate the silicon volume expansions by preparing composites mostly failed to achieve high capacities $\left(>1000 \mathrm{mAhg}^{-1}\right)$. As a consequence, limited amounts of silicon (below 5\%) are currently used to formulate the most advanced industrial silicon-graphite composite electrodes using $\mathrm{SiO}_{x}$ materials [1].

Recently, the introduction of another chemical element in the silicon structure appeared as a promising alternative to enhance the stability and cyclability of Li-ion batteries, while maintaining a high capacity. Germanium raised a growing interest because of intrinsic properties that are highly appealing in view of energy storage applications [7], despite its lower theoretical capacity (1384 mAh.g$\left.{ }^{1}\right)$ with respect to Silicon (3579 $\left.\mathrm{mAh} . \mathrm{g}^{-1}\right)$. Indeed, the electronic and ionic conductivities of germanium exceed by several orders of magnitudes those of Silicon, due to its rather small band gap ( $\sim .6 \mathrm{eV}$, against $1.12 \mathrm{eV}$ for Silicon). Moreover, isotropic lithiation favoring stability is expected in germanium (while it is anisotropic in silicon) due to much facilitated lithium-ion diffusion [8]. In addition, $\mathrm{Si}_{100-x} \mathrm{Ge}_{\mathrm{x}}$ solid solutions can be formed, which may open new perspectives for designing high performance anodes. Silicon-germanium alloys were initially studied mainly for thermoelectric applications [9-12] or as semiconductors [13-16]. Their use as active phase in Li-ion batteries anodes was contemplated only recently. A range of alloys morphologies, e.g. nanofilms, nanowires[17, 18], porous structures [19] and particles $[20,21]$ were synthesized and tested as active materials in Li-lon coin cells. By alloying germanium with silicon in nanofilms, Abel et al were able to increase the capacity of devices [22]. An optimal trade-off between capacity (provided by silicon) and stability (provided by germanium) was found, as the obtained $\mathrm{Si}_{100-x} \mathrm{Ge}_{\mathrm{x}}$ alloys showed better stability than pure silicon and higher capacity than pure germanium [20]. Ge et al used the laser pyrolysis method to produce nanoparticles which exhibited a better capacity retention than micrometric particles [20, 21]. Finally, Kim et al synthesized a core shell nanowire composed of a SiGe core and a Si shell [23]. This material demonstrated improved capacity retention and rate capability as compared to SiGe nanowires [23]. The core shell structure was obtained in a two steps process by migration of a fraction of Silicon atoms to the surface during annealing treatment under reducing atmosphere. The improved performance was attributed to the presence of the Si-rich shell, but the underlying mechanisms remain to be fully elucidated [23].

Here, we have combined the advantages of nanoparticles and core@shell structures by synthesizing core-shell SiGe/Si nanoparticles in one step by laser pyrolysis. Using this method, we have 
achieved a fine control of the particles formation and growth, and produced novel materials composed of a Si-Ge core and a Si-rich shell. The impact of experimental conditions on the particles structure and targeted functionalities was investigated by means of X-ray diffraction (XRD), Raman spectroscopy, electron microscopy, operando synchrotron wide angle $X$-ray scattering, and electrochemical characterizations. We found that the SiGe alloys possess excellent electrochemical stability and performances, outperforming all types of analogous materials [17, 20, 21].

\section{Results and discussions}

The laser pyrolysis method was used to synthetize SiGe nanoparticles, as well as pure silicon and pure germanium reference materials. A schematic view of the laser pyrolysis setup is displayed in Figure S1. Details on the experimental conditions are provided in the experimental part. Materials with different Ge contents, from 0 to $100 \%$, were synthesized by varying the gas ratio of silane vs germane.

The minimum production rate was $3 \mathrm{~g} \cdot \mathrm{h}^{-1}$ when only silane was used (silicon reference). It increased up to $8 \mathrm{~g} . \mathrm{h}^{-1}$ for the production of Ge reference. Assuming 100\% conversion efficiency from $\mathrm{SiH}_{4}$ and $\mathrm{GeH}_{4}$ precursors, production rates of $3.5 \mathrm{~g} \cdot \mathrm{h}^{-1}$ and $9 \mathrm{~g} \cdot \mathrm{h}^{-1}$ were expected. Comparison of calculated and experimental production rates shows that the yield of the reaction was in the range of $85-90 \%$, illustrating the good efficiency of the laser pyrolysis method. Regarding $\mathrm{Si}_{100-x} \mathrm{Ge}_{\mathrm{x}}$ alloys, the production rates were between 4.5 and $7.9 \mathrm{~g} . \mathrm{h}^{-1}$. The values increase with increasing Ge content, due to the higher molar mass of Ge compared to $\mathrm{Si}$. The collected batches were all in gram quantities and experiments were voluntarily stopped when the amount of samples was sufficient for characterizations and electrochemical tests. This production route seems quite advantageous compared to other synthesis methods such as ball-milling, hydrothermal synthesis (batch syntheses), or chemical vapor deposition (low amounts).

Table 1: Main characteristics of produced nanoparticles. Laser power (1050 W) and pressure (atmospheric pressure) were kept constant for the alloys synthesis. Elemental concentration in the powder was measured using EDX at different locations on the sample deposited on a TEM grid. The diameter of the nanoparticles was calculated from Specific Surface Area measurement (see experimental section).

\begin{tabular}{|c|c|c|c|c|}
\hline $\begin{array}{l}\text { Sample } \\
\text { type }\end{array}$ & $\begin{array}{l}\mathrm{GeH}_{4} \text { fraction } \\
\text { in gas } \\
\text { mixture [\%] }\end{array}$ & $\begin{array}{l}\text { at } \% \text { of Ge in } \\
\text { powder [vs Si] }\end{array}$ & $\begin{array}{l}\text { Material } \\
\text { Composition } \\
\text { (EDX) }\end{array}$ & $\begin{array}{c}\text { BET } \\
\text { diameter } \\
\text { [nm] }\end{array}$ \\
\hline Si & $0 \%$ & $0 \%$ & $\mathbf{S i}$ & 71 \\
\hline \multirow{3}{*}{$\begin{array}{l}\text { SiGe } \\
\text { alloys }\end{array}$} & $19 \%$ & $20 \%$ & $\mathrm{Si}_{80} \mathrm{Ge}_{20}$ & 28 \\
\hline & $49 \%$ & $47 \%$ & $\mathrm{Si}_{53} \mathrm{Ge}_{47}$ & 70 \\
\hline & $84 \%$ & $77 \%$ & $\mathrm{Si}_{23} \mathrm{Ge}_{77}$ & 106 \\
\hline Ge & $100 \%$ & $100 \%$ & $\mathrm{Ge}$ & 75 \\
\hline
\end{tabular}

In Table 1, we report the fraction of germane present in the gas mixture introduced in the reactor, as well as the resulting germanium concentration measured in the powders for pure $\mathrm{Si}(0 \% \mathrm{Ge})$, pure Ge $(100 \% \mathrm{Ge})$ and three SiGe alloys with increasing amounts of $\mathrm{Ge}(20,47$ and 77 at\%). The straightforward correspondence between gas fraction and material composition highlights the fine control of the alloy composition using laser pyrolysis. The results were also confirmed by Raman analysis performed on the various samples (Figure 1). The measured spectra are very similar to those previously obtained on particles prepared in the same range of chemical composition [20]. The spectra show a progressive evolution from pure $\mathrm{Si}\left(521 \mathrm{~cm}^{-1}\right)$ to pure $\mathrm{Ge}\left(298 \mathrm{~cm}^{-1}\right)$, with progressive growth and successive disappearance of the Si-Ge bonding $\left(385 \mathrm{~cm}^{-1}\right)$. The maximum relative intensity of the $\mathrm{Si}-\mathrm{Ge}$ bonding is observed for the $\mathrm{Si}_{53} \mathrm{Ge}_{47}$ sample. Moreover, additional information on the 
organization of the material can be accessed by analyzing the Raman peaks position and shape. The spectrum of pure silicon clearly shows a shoulder in the low frequency region, which is usually interpreted as the contribution of an amorphous Si phase [24, 25]. Interestingly, in both $\mathrm{Si}_{80} \mathrm{Ge}_{20}$ and $\mathrm{Si}_{53} \mathrm{Ge}_{47}$ samples, the $\mathrm{Si}$ band is quite broad and positioned below $490 \mathrm{~cm}^{-1}$, therefore indicating a large contribution of an amorphous Si phase. Quite similarly, in these two samples the Ge band is found to be large and shifted towards lower frequencies in comparison with the spectrum of pure Ge. Overall, the $\mathrm{Si}_{80} \mathrm{Ge}_{20}$ alloy is the sample characterized by the broadest full width at half maximum for both $\mathrm{Si}$ and Ge peaks.

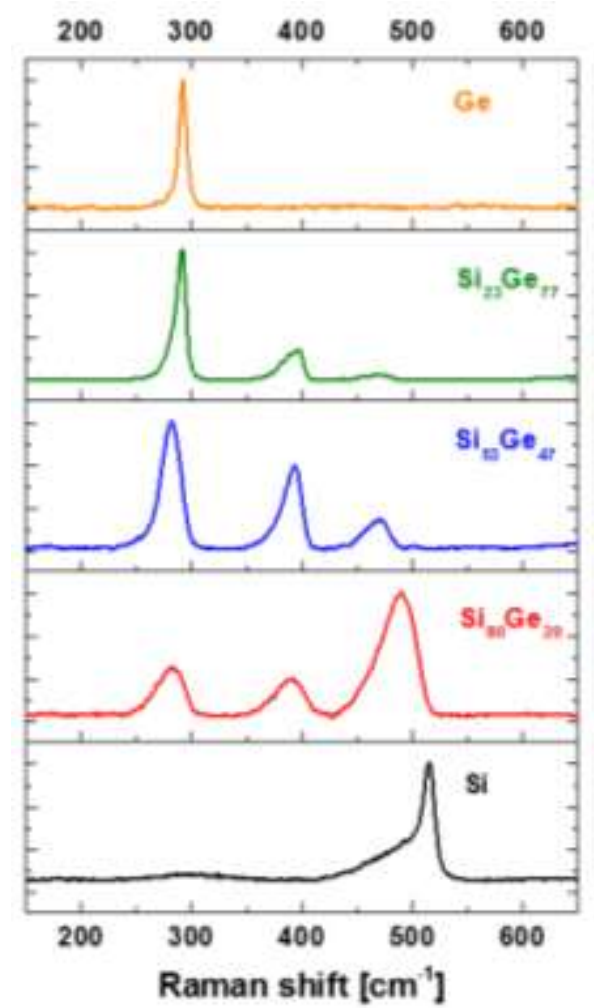

Figure 1: Raman spectra of the various $\mathrm{Si}_{100-x} \mathrm{Ge}_{\mathrm{x}}$ samples, from $\mathrm{x}=100$ (pure silicon) to $\mathrm{x}=0$ (pure germanium).

The morphology of the $\mathrm{Si}_{100-x} \mathrm{Ge}_{x}$ particles was studied by Transmission Electronic Microscopy (TEM) (see typical imaged in SI, Figure S2) and Scanning Transmission Electron Microscopy (STEM), coupled with Energy Dispersive X-ray spectroscopy (EDX) (Figure 2). Note that a small amount of pure Silicon nanoparticles is observed in these pictures. This could be explained by partial decomposition and nucleation from excited silane molecules [26] before thermalization of the reactive medium by collisions. These Si NPs appear as a very minor phase and exhibit sizes in the range 5 to $20 \mathrm{~nm}$, therefore below that of the alloyed Si-Ge particles. They are expected to contribute to the lithiation mechanism, however to an extent that can be neglected at first approximation due to their limited volumic fraction inside the electrodes. The size distributions obtained from TEM analysis (Figure 2d) revealed an average diameter of $27 \pm 9 \mathrm{~nm}\left(\mathrm{Si}_{80} \mathrm{Ge}_{20}\right), 74 \pm 17 \mathrm{~nm}\left(\mathrm{Si}_{53} \mathrm{Ge}_{47}\right)$ and $104 \pm 30 \mathrm{~nm}\left(\mathrm{Si}_{23} \mathrm{Ge}_{77}\right)$, in good agreement with the average diameter deduced from specific surface area measurements (Table 1). The increased average particles size with increased Ge content can be explained considering the values of the melting points, which directly impact the nanoparticles growth mechanism. The melting temperature of $\mathrm{Ge}$ is $1211 \mathrm{~K}$, much lower than that of $\mathrm{Si}(1687 \mathrm{~K})$ [27]. All $\mathrm{Si}_{100-x} \mathrm{Ge}_{\mathrm{x}}$ alloys will melt at 
an intermediate temperature between the two pure compounds reference values. Therefore, the higher the Ge content, the lower the melting temperature. Consequently, larger nanoparticles are expected to be formed at high Ge content as a result of longer favorable coalescence conditions, in agreement with our observations [28].

(a)
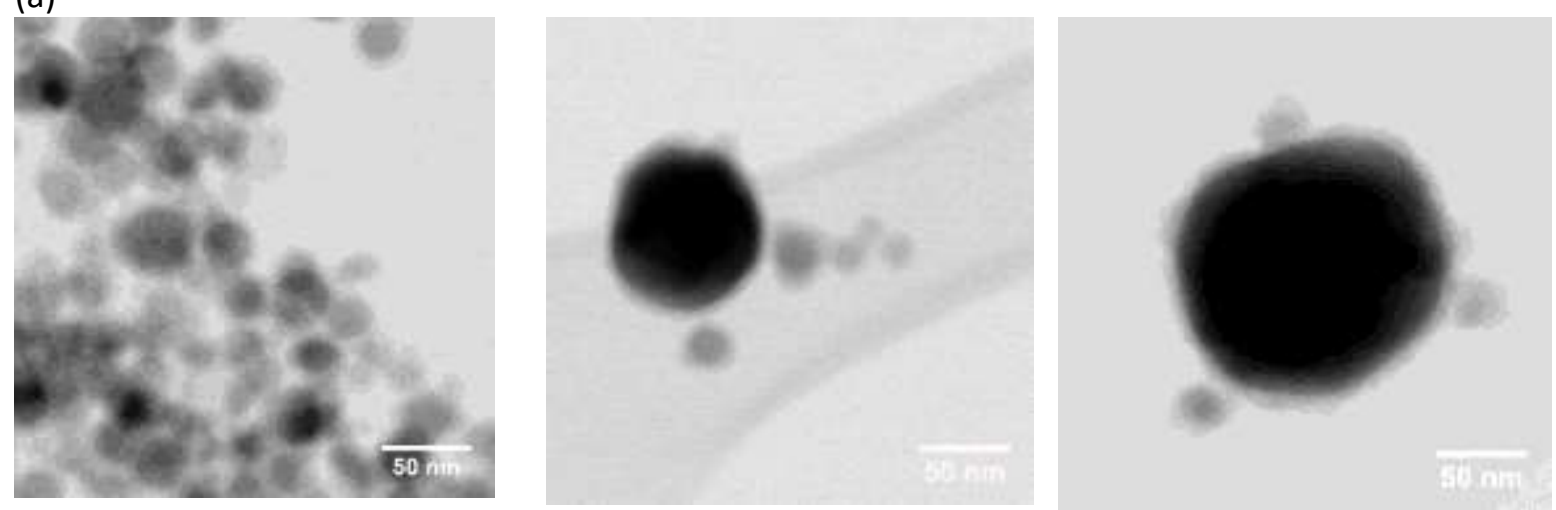

(b)
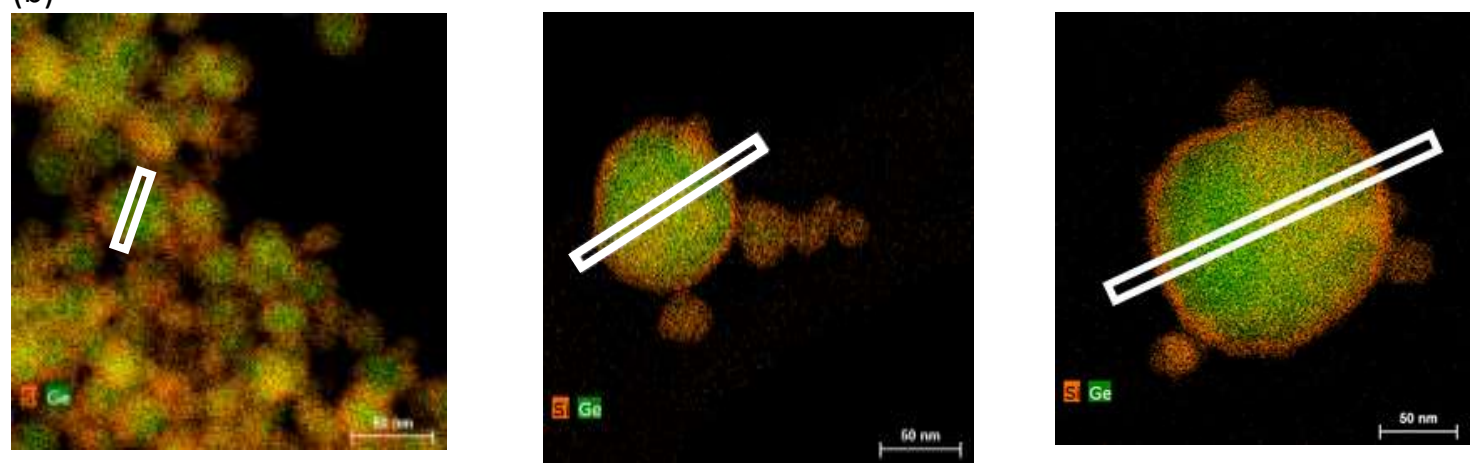

(c)

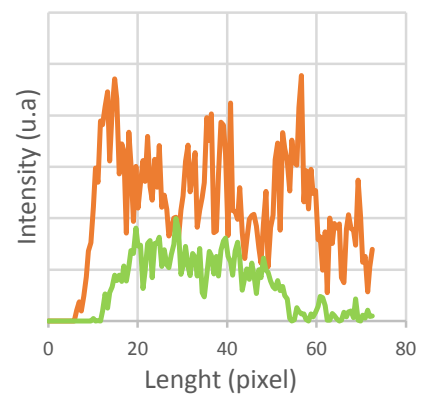

(d)
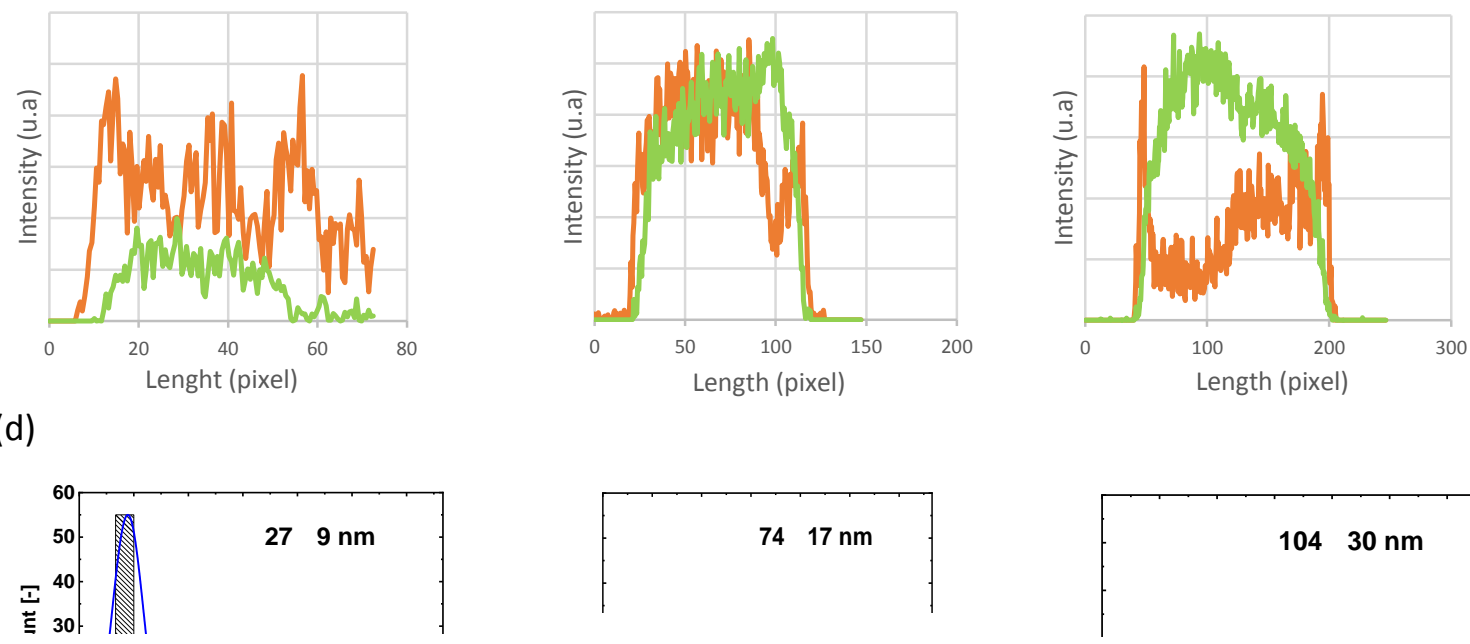

$74 \quad 17 \mathrm{~nm}$

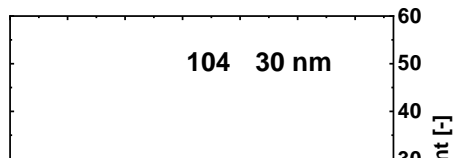

Figure $2:$ a) STEM and b) STEM-EDX pictures of three $\mathrm{Si}_{100-x} \mathrm{Ge}_{x}$ alloys: $\mathrm{Si}_{80} \mathrm{Ge}_{20}$ (left column), $\mathrm{Si}_{53} \mathrm{Ge}_{47}$ (middle column) and $\mathrm{Si}_{23} \mathrm{Ge}_{77}$ (right column), green color corresponds to Ge atoms while orange color corresponds to silicon. The white box on the STEM-EDX pictures corresponds to the position where EDX profiles are obtained as plotted in (c). c) Silicon (orange color) and Ge (green color) 
content across one particle, the $\mathrm{x}$ axis has been adapted for the best visualization of one particle diameter. In each sample, the intensity of orange and blue lines is correlated to the Ge and Si atomic content. d) Average size distribution, obtained by statistical analysis of several TEM images (minimum 100 particles counted).

The internal structure of the $\mathrm{Si}_{100-\mathrm{x}} \mathrm{Ge}_{\mathrm{x}}$ particles can be visualized in the STEM-EDX pictures (Figure $2 \mathrm{~b}$ and $2 c$ ), which reveal the presence of a core shell morphology. The core shell morphology can also be seen by TEM microscopy (see examples in Figure S3). The core of the particles is composed of a silicongermanium alloy (Ge: green, Si: red) while the shell is a silicon-rich phase (red), which is present even at low silicon content ( $\mathrm{Si}_{23} \mathrm{Ge}_{77}$, Figure $2 \mathrm{~b}$, right column). It can be noticed that the core of the SiGe particles is not completely homogeneous at the local scale, as clearly illustrated by the EDX profiles presented in Figure 2c.

Such core shell structure was not observed previously in SiGe particles [20,21, 29], even when the laser pyrolysis method was employed [21]. The peculiar morphologies we obtained may originate from distinct experimental conditions. In their previous work, Ge et al employed a low laser power used with $\mathrm{SF}_{6}$ gas as a sensitizer to heat the reactive mixture by collisional transfer from excited $\mathrm{SF}_{6}$ molecules. However, the temperature of the reactive medium - which is a critical parameter - was not indicated. In our case, flame temperatures were measured in the middle of the reaction flame (Figure 3b) using CARS (Coherent Anti-Stokes Raman Spectroscopy) (see text in SI and figures S4, S5). The flame temperatures were deduced from the population in rotational levels of $\mathrm{H}_{2}$ molecules issued from $\mathrm{SiH}_{4}$ dissociation at three different $\mathrm{He}$ to $\mathrm{SiH}_{4}$ ratios (5, 20 and 40) [30] (Figure 3a). The silane dilution ratio $\left(\mathrm{He} / \mathrm{SiH}_{4}\right)$ corresponding to the synthesis of $\mathrm{Si}_{80} \mathrm{Ge}_{20}, \mathrm{Si}_{53} \mathrm{Ge}_{47}$ and $\mathrm{Si}_{23} \mathrm{Ge}_{77}$ were 15, 20, and 33. The temperatures (deduced from Figure 3a) were found to be respectively $1743 \mathrm{~K}, 1483 \mathrm{~K}$, and $1413 \mathrm{~K}$. According to the phase diagram of $\mathrm{Si}_{100-\mathrm{x}} \mathrm{Ge}_{\mathrm{x}}$ each flame temperature remains above the domain of stability of the related alloy composition (i.e. 1550, 1390 and $1265 \mathrm{~K}$ respectively) [27]. Therefore, the conditions for segregation were always achieved in our experiment, allowing the appearance of the shell.
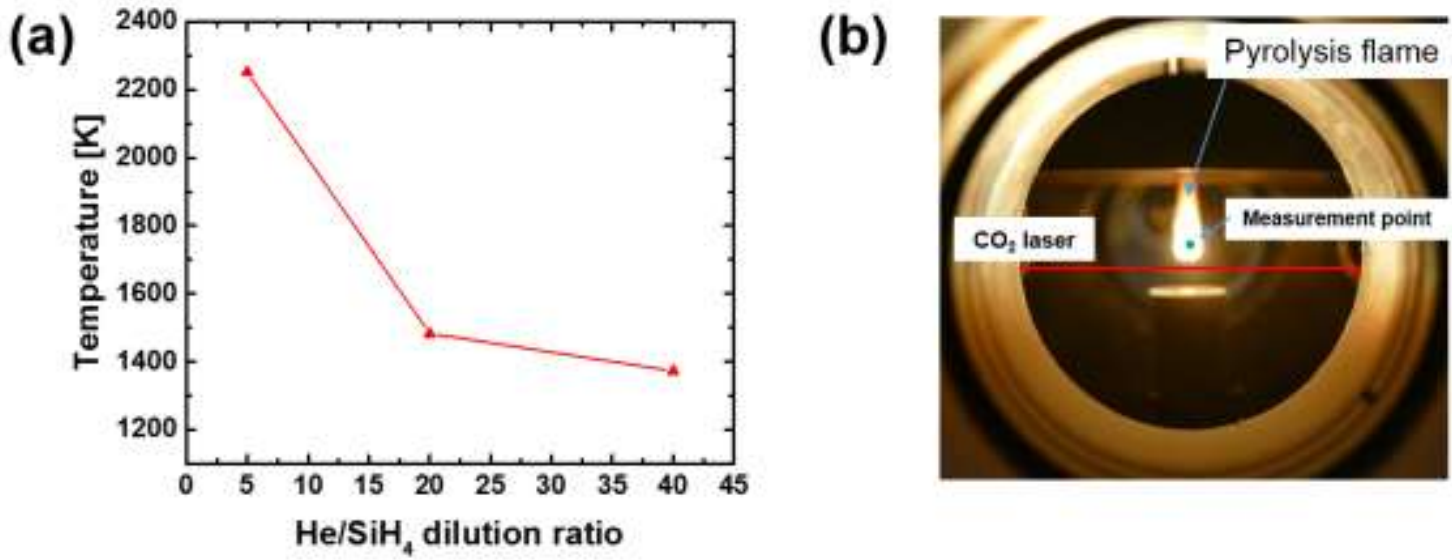

Figure 3: (a) Temperature measurement obtained by CARS spectroscopy during laser pyrolysis of Silane as a function of dilution rate $\left(\mathrm{He} / \mathrm{SiH}_{4}\right)$ and at $1050 \mathrm{~W}$ laser power (b) measurement points taken at $7 \mathrm{~mm}$ above the inlet

Another key factor controlling the synthesis is the atmosphere in which the particles are formed. A $\mathrm{SiGe/Si}$ core shell organization was previously obtained in a nanowire structure after a multi-steps 
treatment [23]. The structure was achieved using a nanowire with homogeneous SiGe composition annealed at $850^{\circ} \mathrm{C}$ in a $\mathrm{H}_{2}$ atmosphere. The reducing atmosphere favored silicon migration to the surface [23]. In our experiment, the nanoparticles were grown in an atmosphere composed of $\mathrm{He}$ and $\mathrm{H}_{2}$, the latter being issued from the decomposition of $\mathrm{SiH}_{4}$ and $\mathrm{GeH}_{4}$. The time of residence in the hot zone was much shorter ( $10^{-3}$ sec compared to 1 hour) but the temperature was quite higher, hence enabling faster kinetics and, potentially, the preferred formation of core-shell structure due to reducing atmosphere.

Insights into the crystalline structure of the particles were further gained by $\mathrm{x}$-ray diffraction (XRD). Xray data obtained on the three SiGe powder samples display diffraction peaks characteristic of the expected diamond structure. In the high Ge content compounds $\mathrm{Si}_{53} \mathrm{Ge}_{47}$ and $\mathrm{Si}_{23} \mathrm{Ge}_{77}$, the peak shape indicates the presence of heterogeneities in $\mathrm{Si}_{100-x} \mathrm{Ge}_{x}$ composition. The data were analyzed by Rietveld structure refinement, assuming the coexistence of several diamond-type crystalline phases with different lattice parameters and different $\mathrm{Si} / \mathrm{Ge}$ compositions. The refinement was obtained by an iterative process: the Ge content was deduced from the lattice parameter value using Vegard's law; the site occupancy for Ge and Si atoms was then fixed accordingly, and the refinement was repeated until convergence was reached. The germanium rich $\mathrm{Si}_{23} \mathrm{Ge}_{77}$ compound exhibits strong structural composition heterogeneities. Four distinct phases were needed to account for the peak shape (fig. S6). The $\mathrm{Ge}$ content values deduced from the refined lattice parameters were found to range from $x=100$ (pure $\mathrm{Ge}$ ) to $\mathrm{x}=70$. Considering the relative crystalline phase amounts, this corresponds to a mean $\mathrm{Ge}$ content $\langle x\rangle=79 \pm 5$, in very good agreement with the value determined by EDX. Regarding $\mathrm{Si}_{53} \mathrm{Ge}_{47}$, two different alloy phases had to be taken into account, with respective germanium contents $x=65$ and $x=50$ in relative amounts 1:4 (figure 4). This results in a mean Ge content $\langle x\rangle=53 \pm 5$ which is close to but significantly different from the composition determined by EDX. By contrast, the $\mathrm{Si}$ rich $\mathrm{Si}_{80} \mathrm{Ge}_{20}$ compound appears to be more homogeneous in composition. However, as for the $\mathrm{Si}_{53} \mathrm{Ge}_{47}$ alloy, the XRD-determined mean Ge content $\langle x\rangle=40$ is higher than the EDX result. Moreover, this sample is characterized by a strong strain as evidenced by the rapid peak width increase upon increasing $2 \theta$, and a lower crystallinity than the two other Ge rich compounds. This observation might explain the discrepancies in composition found in the two Si rich alloys by XRD and EDX as the former strictly corresponds to the crystalline component, while the latter averages both crystalline and amorphous components over the whole sample. The XRD results are consistent with the Raman data (Figure 1) as the silicon peak for the $\mathrm{Si}_{80} \mathrm{Ge}_{20}$ compound was found to be broader and shifted towards smaller wavenumbers, which indicated a more amorphous silicon phase. To complement the powder results, measurements were performed on the electrodes elaborated from these samples. Similar results were obtained (figure $\mathrm{S} 7 \mathrm{in} \mathrm{SI}$ ) confirming that, as expected, the electrode manufacturing process did not modify the structure of the material. 


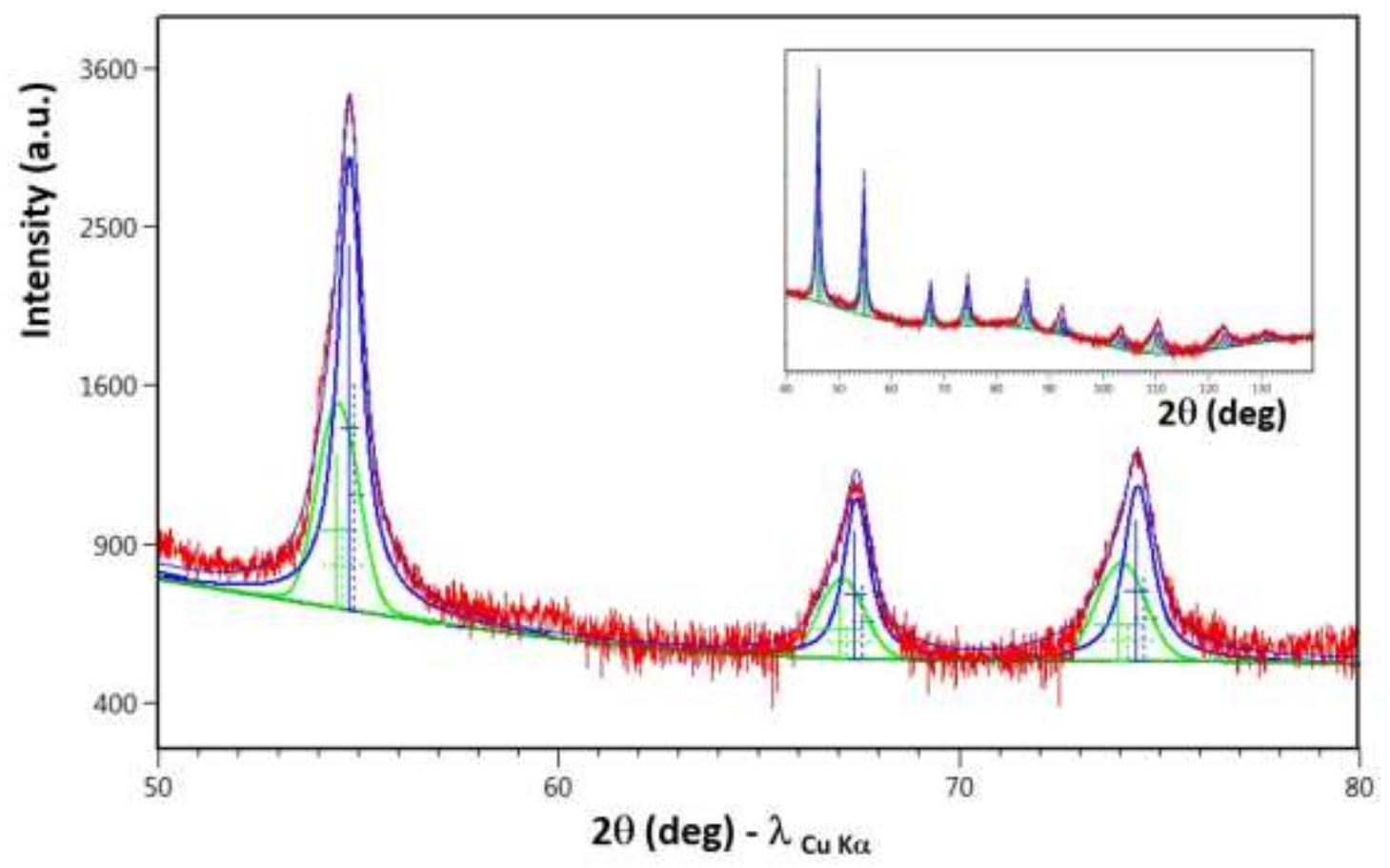

Figure 4 : X-ray diffraction peaks of $\mathrm{Si}_{53} \mathrm{Ge}_{47}$ powder (red: experimental data - black: result of the Rietveld structure refinement considering two diamond-type crystalline phases to account for the chemical composition inhomogeneities - green and blue: contributions of the as determined $\mathrm{Si}_{0.35} \mathrm{Ge}_{0.65}$ and $\mathrm{Si}_{0.5} \mathrm{Ge}_{0.5}$ phases). The inset shows full experimental and refined diffractograms.

At this stage, in order to investigate the (de)alloying properties of the best performing SiGe compounds (see electrochemical results), operando synchrotron WAXS measurements were performed on $\mathrm{Si}_{53} \mathrm{Ge}_{47}$ alloy electrodes assembled in coin cells vs lithium. The results are displayed on Figure 5. 


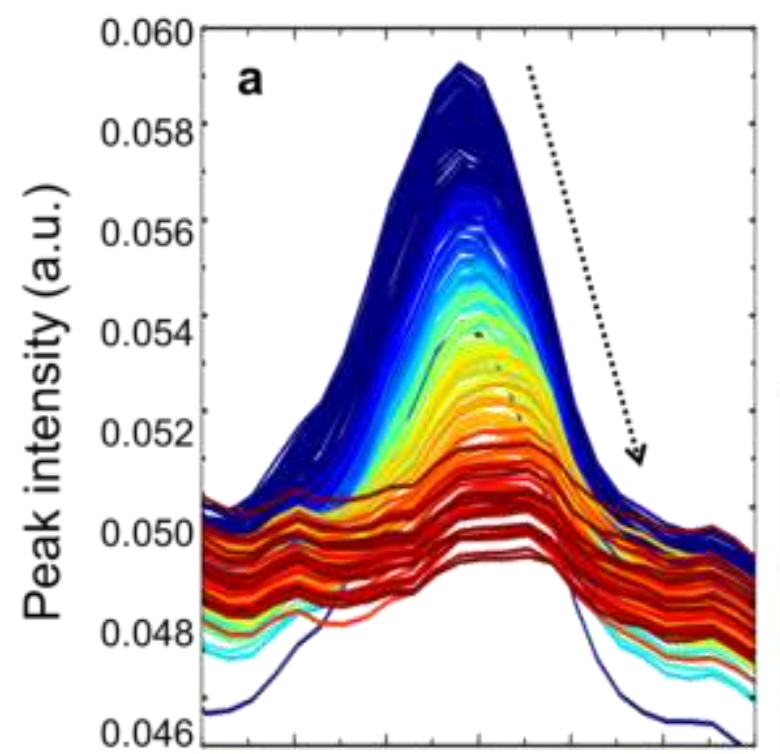

$\begin{array}{lllllll}1.90 & 1.92 & 1.94 & 1.96 & 1.98 & 2.00 & 2.02\end{array}$ $\mathrm{Q}\left(\AA^{-1}\right)$

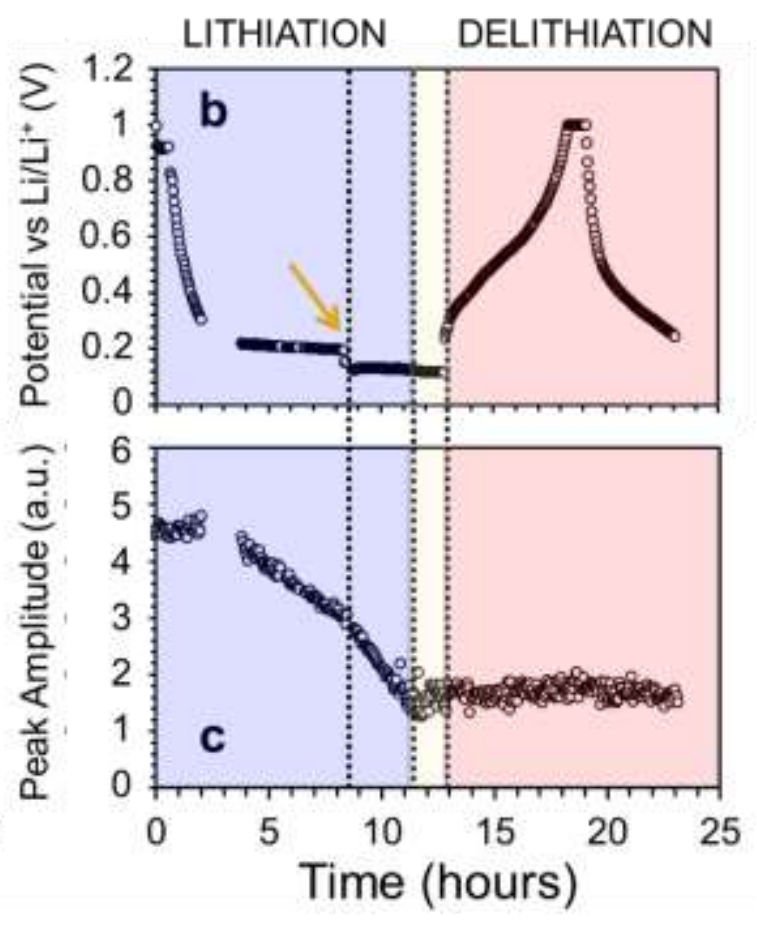

Figure 5 : a) Si-Ge [111] peak intensity variations measured by operando synchrotron XRD during the first lithiation. The peak decreases in intensity (from blue to red) indicating the continuous amorphization of the active particles. b) Voltage against time, with a change in current at ca. 8 hours, indicated by the arrow, and a floating period at the end of the lithiation, materialized by the yellow shadowed area. c) Peak amplitude obtained by a Gaussian fit to the XRD data, as a function of time.

The time dependency of the cell potential along the first cycle is reported on Figure $5 \mathrm{~b}$. The cell was cycled at a rate of $\mathrm{C} / 20(\mathrm{I}=-80 \mu \mathrm{A})$ during $8 \mathrm{~h}$. At that point, the current was increased up to $240 \mu \mathrm{A}$ to accelerate the lithiation process. The diffraction patterns composed of Bragg refflections from the battery components ( $\mathrm{Cu}$ and $\mathrm{Al}$ current collectors, Si-Ge particles) were measured every two minutes. Figure 5 a) shows the evolution of the SiGe (111) peak upon time during the first lithiation. As the low Q-resolution associated to the experimental operando WAXS conditions did not allow to describe the asymmetrical $\mathrm{Si}_{100-x} \mathrm{Ge}_{x}$ diffraction peak shape, the SiGe (111) peak was fitted considering a single Gaussian function to obtain the time (or, equivalently, voltage) dependency of the intensity (Figure S8 in $\mathrm{SI}$ ). We observed that, during the first lithiation, the Bragg reflection of SiGe linearly decreased in intensity, as already observed in pure Silicon nanoparticles based anodes by Tardif et al.[31], reflecting the continuous amorphization of crystalline particles due to the alloying process. The in situ WAXS data therefore confirm that full amorphization is achieved in the Si-Ge compound, i.e. the structural and chemical heterogeneities of the core-shell alloyed particles do not impede the lithium to penetrate to the core of the particles during lithiation. Note that there is a slope variation in the integrated intensity (indicated by the yellow arrow in Figure $5 c$ ) due to the corresponding increase in the current at time $\sim 8$ hours (Voltage $=0.19 \mathrm{~V}$ ). A small broad bump visible at the end of the first lithiation and of constant intensity can be observed during the floating period (shadowed in yellow on Figures $5 b, c$ ) and the delithiation step. As this broad feature is not present for the other SiGe diffraction peaks, it may be considered as a background contribution.

Along the lithiation process, the diffraction peak continuously shifted in position towards higher $Q$ values. This evolution might originate, at least partially, from the sequential lithiation of the different $\mathrm{Si}_{100-x} \mathrm{Ge}_{x}$ phases depending on the Ge content. Indeed, as $\mathrm{Ge}$ and $\mathrm{Si}$ have distinct lithiation potential $\mathrm{E}$ 
vs $\mathrm{Li}^{+} / \mathrm{Li}$ (respectively close to $380 \mathrm{mV}$ and $250 \mathrm{mV}$ ) [32], Ge-rich phases are expected to lithiate first. Another possible mechanism leading to Bragg peak displacement could be the increasing compressive stress applied to the core of the nanoparticles by the lithiation front trapped between the pristine core and the outer lithiated layer, as already observed in pure Si nanoparticles [31, 33]. Both phenomena could induce the Q-shift. High resolution in situ data would be needed to assess the possible mechanisms and possibly quantify them.

At this point, we have characterized the nanoparticles in terms of average size, internal structure, elemental composition and crystalline phases, and we have evidenced their complete amorphization during the first lithiation. Common features to all $\mathrm{Si}_{100-x} \mathrm{Ge}_{x}$ are the core shell morphology and diamondtype crystalline structure. The mean particle size and degree of structural heterogeneity were found to depend on the Ge content. Following these physical characterizations, we evaluated the electrochemical performance by cycling the three SiGe electrodes assembled in half-cells, and compared their behavior to reference Si and Ge based batteries.

The specific charge capacities of the five materials obtained for the first sixty cycles are shown on Figure 6.a. As expected, the cell capacity is globally decreased when the amount of germanium is increased. Interestingly, it also becomes more stable, i.e. the capacity retention is improved when alloying silicon with germanium. In case of pure silicon, the capacity drops from $3217 \mathrm{mAh} . \mathrm{g}^{-1}$ to $2394 \mathrm{mAh} . \mathrm{g}^{-1}$, which corresponds to a capacity retention of $74 \%$. In the $\mathrm{Si}_{100-x} \mathrm{Ge}_{\mathrm{x}}$ alloys with $\mathrm{x}=20$ and $x=47$, the capacity decreases from $2373 \mathrm{mAh} \cdot \mathrm{g}^{-1}$ to $1958 \mathrm{mAh} \cdot \mathrm{g}^{-1}$ (84\% retention), and from 1877 mAh.g $\mathrm{g}^{-1}$ to 1695 mAh.g ${ }^{-1}$ (90\% retention), respectively. In $\mathrm{Si}_{23} \mathrm{Ge}_{77}$ the specific charge capacity decreases from $1435 \mathrm{mAh} . \mathrm{g}^{-1}$ to $1275 \mathrm{mAh} . \mathrm{g}^{-1}$ (89\% retention). In pure Ge, the capacity fades from $1103 \mathrm{mAh} . \mathrm{g}^{-1}$ to $1054 \mathrm{mAh} . \mathrm{g}^{-1}$ corresponding to $96 \%$ of capacity retention. These results show that the highest capacity associated with good stability was obtained for $\mathrm{Si}_{53} \mathrm{Ge}_{47}$ material, as was already pointed by other groups [17, 20-22]

(a)

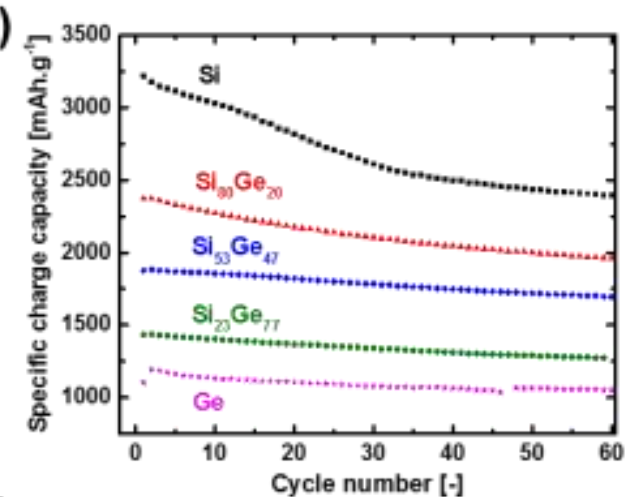

(c)

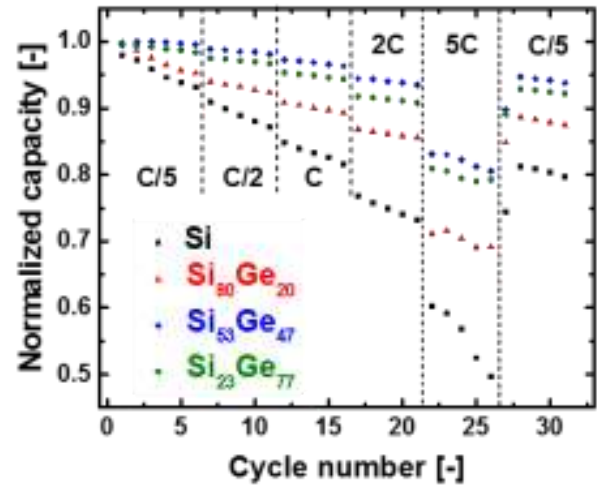

(b)

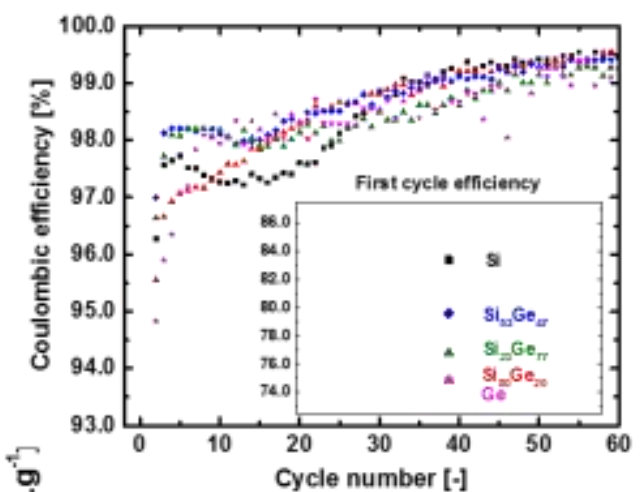

(d)

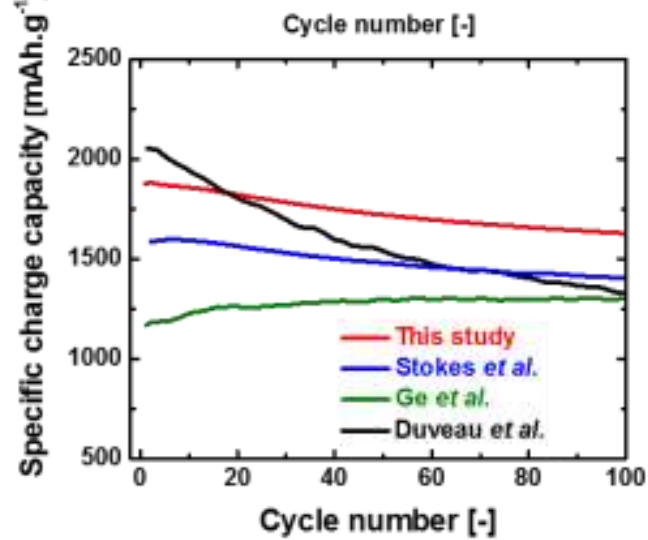


Figure 6: (a) Galvanostatic cycling performances of the alloys compared to silicon. Specific charge capacities are normalized with the mass of active material; (b) coulombic efficiencies of mentioned materials, the inset presents the coulombic efficiencies of first cycles for each material. Ge and $\mathrm{Si}_{80} \mathrm{Ge}_{20}$ dots are overlapped; (c) galvanostatic cycling at increasing current densities; (d) comparison of $\mathrm{Si}_{53} \mathrm{Ge}_{47}$ performance in galvanostatic cycling with other $\mathrm{Si}_{50} \mathrm{Ge}_{50}$ material performance reported in the literature where Stokes et al, Ge et al and Duveau et al correspond to references [17, 21, 20] respectively.

In figure 6.b, the coulombic efficiencies of the various alloys are displayed together with those of pure silicon. All materials, except $\mathrm{Si}_{80} \mathrm{Ge}_{20}$, exhibit qualitatively the same behavior: the coulombic efficiency increases during the first cycles, then decreases at typically cycles 5 to 10 before reaching a plateau up to cycle 20 , followed by a steady increase. In case of the Si-rich alloy $\mathrm{Si}_{80} \mathrm{Ge}_{20}$, the values steadily and continuously increase since the early cycles. As mentioned before, the average size of the SiGe alloy nanoparticles depends on the $\mathrm{Ge}$ content. Hence, as $\mathrm{Si}_{80} \mathrm{Ge}_{20}$ is composed of particles of smaller sizes than the other two alloy materials (Table 1), its contrasting behavior could be explained by the reduced diameter of the particles, which could lead to more SEI formation. Nevertheless, $\mathrm{Si}_{53} \mathrm{Ge}_{47}$ sample exhibits better coulombic efficiencies than pure $\mathrm{Si}$ (which is made of similar particles of comparable size). Therefore, the results cannot be rationalized by considering only size effects. Clearly, the presence of germanium has a positive impact on the electrochemical efficiency during the first cycles.

Additional insights into the alloys properties were gained by analyzing the galvanostatic cycling at higher C-rates (up to $5 \mathrm{C}$ ) shown in Figure $6 \mathrm{c}$. In case of pure silicon, the specific capacity rapidly decreases down to $60 \%$ (beginning of $5 \mathrm{C}$ ) and $50 \%$ (end of $5 \mathrm{C}$ ) of its initial capacity. $\mathrm{Si}_{80} \mathrm{Ge}_{20}$ retains $75 \%$ and $\mathrm{Si}_{53} \mathrm{Ge}_{47}$ exhibits the best performances with $85 \%$ of its initial capacity retained at a current of $5 \mathrm{C}$. We can suppose that the higher conductivity of alloyed materials allows a better diffusivity of lithium, this effect being exacerbated at high germanium contents. The optimal trade-off to best compromise capacity and stability was found for the composition close to 50/50 atomic ratio between silicon and germanium. This material could demonstrate higher specific charge capacity than other types of SiGe materials, as highlighted in Figure 6.d where we have compared its electrochemical behavior to available literature data on similar compounds. Electrode compositions (active loading) and capacities are different however, it is probable that the nanometric size of our particles and the presence of the silicon shell play a key role in improving the performance of these materials when used as negative electrode in half cells. It can be hypothesized that the alloy core stabilizes the capacity, acting as a frame during sequential lithiation and delithiation of the silicon shell [23].

\section{Conclusions}

Using the laser pyrolysis method, we were able to obtain a variety of SiGe/Si core-shell nanoparticles with controlled amounts of germanium varied in the range 20 to 80 at\%. The one-step synthesis was performed with good yields and high production rates. The nanoparticles consist of a Si-rich shell surrounding the alloyed core, a peculiar morphology that was explained by reaction temperature measured at the local scale. Each alloy is composed of a variety of phases with different silicon/germanium ratio and a common diamond structure. Upon electrochemical cycling, the phases are successively lithiated - at least during the first cycle - and remain amorphous afterwards. The electrochemical capacity of all alloy compositions was found to be higher than that of pure germanium, while stability upon cycling was improved compared to pure silicon and much less dependent on the cycling rate. The best performances were obtained for $\mathrm{Si}_{53} \mathrm{Ge}_{47}$ with a reversible capacity of $1685 \mathrm{mAh} . \mathrm{g}^{-1}$ after 60 cycles, a value never attained to date in state-of-the art compounds prepared 
with similar compositions. Moreover, both coulombic efficiencies during the first ten cycles and rate capabilities were significantly improved compared to all other types of SiGe materials. These results demonstrate the capability to outperform actual SiGe systems by designing nanosized objects integrating structural heterogeneities beneficial to lithium diffusion and alloying processes. Further investigations are required to fully understand the (de)lithiation mechanism, in particular the role and evolution of the silicon shell after few cycles, i.e. understand whether it is maintained around the particles during the amorphisation process or evolves towards a homogeneous alloy.

\section{Experimental section :}

- Material synthesis :

Nanosized silicon-germanium, silicon and germanium particles were synthetized by laser pyrolysis. The precursors were: ultra high purity silane ( $99.999 \%$ purity) gas purchased from Praxair, and germane (20 wt\% in $\mathrm{He}$ ) obtained from Air Liquide Deutschland $\mathrm{GmbH}$. Silane and germane precursors were diluted with He (Air Products, BIP ${ }^{\circledR}$ grade, $99.99 \%$ purity). Different gas ratio (silane vs germane) were used to achieve the synthesis of nanoparticles with various Si vs Ge compositions (Table I). Pure silicon and germanium nanoparticles were also synthesized as reference materials. In the latter case, ethylene $\left(\mathrm{C}_{2} \mathrm{H}_{4}\right)$ was used as a sensitizer because germane does not absorb the laser radiation. A small amount of $\mathrm{C}(2.6 \mathrm{wt} \%)$ due to ethylene dissociation is present in the Ge reference. A high power $\mathrm{CO}_{2}$ Laser (PRC SLS 2800) was operated in continuous mode and delivered a $20 \mathrm{~mm}$ diameter beam, the power (measured after the reactor without reactive gases) was fixed at $1050 \mathrm{~W}$. The gas flows were set with mass flowmeters (Brooks Smart flow meter) while the pressure inside the reactor was regulated at 740 Torr. The nanopowders were transferred by an argon flow from the reaction zone to filters where they were stopped and collected.

\section{- Material characterizations :}

TEM images (not shown here) were acquired with a JEOL 2010 High resolution transmission electron microscope at $200 \mathrm{kV}$ and were used for size distribution analysis. Local morphology and STEM-EDX analyses were performed on a FEI Titan Themis (probe corrected) operating at $200 \mathrm{kV}$ and equipped with a High Angle Annular Dark Field (HAADF) Scanning Transmission Electron Microscopy (STEM) module and SuperX EDX detector system. The probe current was $150 \mathrm{pA}$ with a probe size of $0.1 \mathrm{~nm}$ at Full Wave Half-Maximum. EDX acquisition was performed in 20 minutes, and spectra have been deconvoluted by the Cliff-Lorimer method to extract elemental contributions (Si-K $\alpha 1.739 \mathrm{keV}$, Ge-Ka 9.874keV).

The Brunauer, Emmet and Teller (BET) method was used to measure the $S_{B E T}$ specific surface of the different samples using a Micromeritic apparatus (Flowsorb 2300). The diameters of the particles (Table I) were calculated using the formula $D=6000 /$ (density $\times S_{B E T}$ ) assuming a spherical shape of the particles. The density of the different samples was estimated from the composition determined by EDX measurements and assuming a density of 2.33 (He picnometry measurement) for Si and bulk value of 5.32 for Ge. Raman spectra were acquired from Horiba XploRA PLUS with a $532 \mathrm{~nm} \mathrm{Ar}$ laser with a laser power of $0.79 \mathrm{~mW} . \mathrm{cm}^{-2}$.

Powder X-ray diffraction measurements were carried out on a Panalytical X'Pert powder diffractometer equipped with a copper anode $\left(\lambda_{\kappa \alpha 1}=1.5406 \AA, \lambda_{\text {Ka2 }}=1.5444 \AA\right.$ ) and an X'Celerator 1D detector. It was configured in Bragg-Brentano geometry, with a variable divergence slit on the primary beam path and a set of antiscattering slits positioned before and after the sample. Axial divergence was limited by 0.02 rad Soller slits. 
An ink was first prepared to obtain electrodes. Super $\mathrm{P}$ (Timcal) was used as electronic percolator. The binder was Carboxymethylcellulose (DS $=0.7, \mathrm{Mw}=250 \mathrm{~kg} \cdot \mathrm{mol}^{-1}$ ) (Sigma-Aldrich). It was previously diluted at $3 \%$ in deionized water. Slurry of active material was made with a (50/25/25) formulation of (active material/CMC/Carbon SP). Deionized water $(1 \mathrm{~mL})$ was used as the wetting agent and isopropanol as the solvent of the preparation. This slurry was then spread onto a $12 \mu \mathrm{m}$ thick copper foil with a doctor blade coating machine in order to obtain a uniform $100 \mu \mathrm{m}$ layer of ink. The film was further dried and $14 \mathrm{~mm}$ pellets are cut out from the film and pressed at 1 ton before being placed under vacuum at $120^{\circ} \mathrm{C}$ for drying during 2 days.

CR 2032 half-cells batteries were assembled inside an Argon-filled glove-box. Viledon paper was soaked with electrolyte. The electrolyte was composed of $1 \mathrm{M} \mathrm{LiPF}_{6}$ dissolved in EC/DEC (Ethylene carbonate/diethyl carbonate $1: 1, \mathrm{v} / \mathrm{v}$ ) with $2 \%$ VC (vinyl carbonate) and $10 \%$ FEC (fluoro ethyl carbonate). Cellgard was used as separator and Li metal discs as counter electrode.

Cyclic voltammetry (CV) and electrochemical cycling experiments were performed on a VMP-3 system

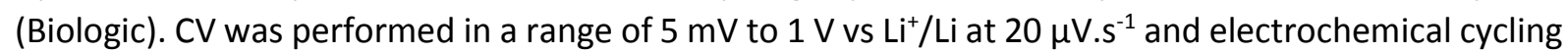
was performed by galvanostatic charge/discharge of the coin-cells in the range of $5 \mathrm{mV}$ to $1 \mathrm{~V} \mathrm{vs} \mathrm{Li}^{+} / \mathrm{Li}^{\text {. }}$

\section{- Operando WAXS}

Operando synchrotron wide-angle X-ray scattering (WAXS) was conducted on the D2AM (BM02) beamline of the European Synchrotron Radiation Facility (ESRF, Grenoble, France). The incident energy was set at $17 \mathrm{keV}$ (incoming wavelength $\lambda=0.7294 \AA$ ) with a beam size at the sample position of $0.3 \times$ $0.3 \mathrm{~mm}^{2}$. The diffracted beam was measured on an imXPAD WOS 2-dimensional detector. The sampleto-detector distance was $20.16 \mathrm{~cm}$ and calibrated using chromium oxide powder. The measured $Q$ values were in the range $\left[0.4-4 \AA^{-1}\right]$, $Q$ being the momentum transfer defined as $Q=\frac{4 \pi \sin \theta}{\lambda}$. The $Q$ resolution is estimated at $0.01 \AA^{-1}$ from the pixel size and detector distance. The WAXS intensity profiles as a function of $Q$ were obtained by azimuthal integration of the 2D patterns using the PyFAl library. The pouch cell was assembled using a $2 \times 2 \mathrm{~cm}^{2}$ square anode containing $0.97 \mathrm{mg} / \mathrm{cm}^{2}$ active material $\mathrm{Si}_{53} \mathrm{Ge}_{47}$, carbonate mixture-based electrolyte and lithium metal as cathode, and mounted in transmission geometry.

\section{Acknowledgements}

This work was funded under H2020 European project SPICY (Project Grant Agreement number: 653373) and French National research agency project HELIOS (ANR 16-CE05-0028-03). The support of CEA Flaghip "operando" and "SiBali" is gratefully acknowledged. STEM measurements were performed at NanoTEM, granted under the program Future Investments" with the reference ANR-10-EQPX-50. Brigitte Tretout from ONERA is acknowledged for her help into issuing the CARS experiments. Nils Blanc, Nathalie Boudet, Gilbert Chahine and Stephan Arnaud from beamline D2AM (BM02) at the ESRF are gratefully acknowledged for their help during the synchrotron experiments as well as ESRF for beam time allocation. The WOS detector used for the synchrotron WAXS measurements was funded by the French National Research Agency (ANR) under the "Investissements d'avenir" program with the grant number: ANR-11-EQPX-0010. 


\section{Keywords}

Anode; Core-shell; Laser pyrolysis; Nanoparticles; SiGe Alloys -

\section{Table of Contents text}

This article shows the first example of one-step synthesis of core shell SiGe@Si nanoparticles by laser pyrolysis. Several $\mathrm{Si}_{100-x} \mathrm{Ge}_{x}, \mathrm{x}=20$ to 80 alloy compositions are synthetized. XRD and WAXS characterizations give a complete picture of the material structure, and the $\mathrm{Si}$ shell is observed whatever the average composition. The $\mathrm{Si}_{53} \mathrm{Ge}_{47}$ composition exhibits the best electrochemical performance reported so far in compounds of similar composition, with a reversible specific capacity of $1695 \mathrm{mAh} . \mathrm{g}^{-1}$ after 60 cycles. 


\section{References}

[1] J. W. Choi, D. Aurbach, Nat. Rev. Mater. 2016, 1 (4), 16013, 1-16

[2] M. Obrovac, V. Chevrier, Chem. Rev. 2014, 114 (23), 11444-11502.

[3] S. H. Ng, J. Wang, D. Wexler, K. Konstantinov, Z. P. Guo, H. K. Liu, Angew. Chem., Int. Ed. 2006, 45 (41), 696-6899.

[4] Y. Hwa, W.S.Kim, W. S. H. Hong, H. J. Sohn, Electrochim. Acta 2012, 71, 201-205.

[5] S. Chen, M. L. Gordin, R. Yi, G. Howlett, H. Sohn, D. Wang, Phys. Chem. Chem. Phys. 2012, 14 (37), 12741-12745.

[6] J. Sourice, A. Quinsac, Y. Leconte, O. Sublemontier, W. Porcher, C. Haon, A. Bordes, E. De Vito, A. Boulineau, S. Jouanneau Si Larbi, N. Herlin-Boime, C. Reynaud, ACS Appl. Mater. Interfaces 2015, 7 (12), 6637-6644.

[7] D. Wang, Y. L. Chang, Q. Wang, J. Cao, D.B. Farmer, R. G. Gordon, H. Dai, J. Am. Chem. Soc 2004, 126 (37), 11602-11611.

[8] W. Liang, H. Yang, F. Fan, Y. Liu, X. H. Liu, J. Y. Huang, T. Zhu, S. Zhang, ACS Nano 2013, 7 (4), 3427-3433.

[9] M. Heller, R. Nasby, R. Johnson Jr, J. Appl. Phys. 1976, 47 (9), 4113-4121.

[10] M. Strasser, R. Aigner, M. Franosch, G. Wachutka, Sens. Actuators, A: Physical 2002, 97, 535542.

[11] N. Mingo, D. Hauser, N. Kobayashi, M. Plissonnier, A. Shakouri, A., Nano Lett. 2009, 9 (2), 711-715.

[12] H. Lee, D. Vashaee, D. Wang, M. S. Dresselhaus, Z. Ren, G. Chen, J. Appl Phys 2010, 107 (9), $094308,1-8$

[13] K. Ismail, J. Chu, B. Meyerson, Appl. Phys. Lett. 1994, 64 (23), 3124-3126.

[14] S. Imai, Y. Hiraoka, A. Kurobe, N. Sugiyama, T. Tezuka, Toshiba Corp, 1998.

[15] J. D. Cressler, IEEE Trans. Microwave Theory Tech. 1998, 46 (5), 572-589.

[16] M.L. Lee, E. A. Fitzgerald, M. T. Bulsara, M. T. Currie, A. Lochtefeld, J. Appl. Phys. 2005, 97 (1), 011101, 1-27

[17] K. Stokes, H. Geaney, G. Flynn, M. Sheehan, T. Kennedy, K.M. Ryan, ACS Nano 2017, 11 (10), 10088-10096.

[18] T. Kennedy, M. Brandon, K.M. Ryan, Adv. Mater. 2016, 28 (27), 5696-5704.

[19] J. Wang, N. Du, Z. Song, H. Wu, H. Zhang, D. Yang, J. Power Sources 2013, 229, 185-189.

[20] D. Duveau, B. Fraisse, F. Cunin, L. Monconduit, Chem. Mater. 2015, 27 (9), 3226-3233.

[21] M. Ge, S. Kim, A. Nie, R. Shahbazian-Yassar, M. Mecklenburg, Y. Lu, X. Fang, C. Shen, J. Rong, S. Y. Park, Nanotechnology 2015, 26 (25), 255702, 1-9

[22] P. R. Abel, A. M. Chockla, Y. M. Lin, V. C. Holmberg, J. T. Harris, B. A. Korgel, A. Heller, C. B. Mullins, ACS Nano 2013, 7 (3), 2249-2257.

[23] H. Kim, Y. Son, C. Park, M. J. Lee, M. Hong, J. Kim, M. Lee, J. Cho, H. C. Choi, Nano Lett. 2015, $15(6), 4135-4142$.

[24] I. Campbell, P. M. Fauchet, Solid State Commun. 1986, 58 (10), 739-741.

[25] V. Paillard, P. Puech, M. Laguna, R. Carles, B. Kohn, F. Huisken, J. Appl. Phys. 1999, 86 (4), 1921-1924.

[26] W. R. Cannon, S.C. Danforth, J. H. Flint, J. S. Haggerty, R. A. Marra, J. Am. Ceram. Soc. 1982, 65(7), 324-330

[27] R. Olesinski, G. Abbaschian, J. Phase Equilib. 1984, 5 (2), 180-183.

[28] S.E. Pratsinis, S. Vemury, Powder Technol. 1996, 88 (3), 267-273.

[29] C. Mehringer, C. Kloner, B. Butz, B. Winter, E. Spiecker, W. Nanoscale 2015, 7 (12), 51865196.

[30] F. Lacour, O. Guillois, X. Portier, H. Perez, N. Herlin, C. Reynaud, Phys. E 2007, 38 (1-2), 11-15.

[31] S. Tardif, E. Pavlenko, L. Quazuguel, M. Boniface, M. Maréchal, J.-S. Micha, L. Gonon, V. Mareau, G. Gebel, P. Bayle-Guillemaud, ACS Nano 2017, 11 (11), 11306-11316. 